\title{
Cyclodextrin short-nanofibers using sacrificial electrospun polymeric matrix for VOC removal
}

\author{
Asli Celebioglu ${ }^{1} \cdot$ Tamer Uyar $^{1} \mathbb{D}$
}

Received: 16 May 2017 / Accepted: 11 November 2017 / Published online: 18 November 2017

○) Springer Science+Business Media B.V., part of Springer Nature 2017

\begin{abstract}
Cyclodextrins (CD) are cyclic oligosaccharides that can form noncovalent host-guest inclusion complexes to yield intriguing supramolecular structures. Electrospinning of nanofibers from CD is challenging since they are small molecules, nonetheless, electrospun nanofibers from CD would be particularly attractive because of the distinctive properties obtained by combining the very large surface area of nanofibers along with the inclusion complexation capability of CD. Herein, we performed the electrospinning of native $\mathrm{CD}$ type (i.e. $\gamma$-CD) using a minimal amount of carrier polymeric matrix (polyethylene oxide (PEO)). Once, the uniform nanofibers were electrospun from $\gamma$-CD/PEO systems, the polymeric carrier matrix was selectively removed by simple washing procedure, at the end, $\gamma$-CD short-nanofibers were obtained. We observed that $\gamma$-CD short-nanofibers could remove volatile organic compounds (VOC) (i.e. aniline) due to the inclusion complexation capability whereas pristine $\gamma$-CD powder could not have the capability for the VOC removal.
\end{abstract}

Keywords Electrospinning $\cdot$ Nanofibers $\cdot$ Cyclodextrin $\cdot$ VOC removal

\section{Introduction}

Electrospinning has become a widely used technique in academia as well as in industry for the production of nanofibers due to its simplicity, versatility and cost-effectiveness [1]. Electrospun nanofibers/nanowebs have very high surface area, nanoporous feature, and structural design flexibility, therefore, they are quite applicable in various fields including filtration, biotechnology, energy, sensors, catalysis, electronics, textile, food, packaging, agriculture, etc [1-4]. Due to their exceptional properties, electrospun nanofibers/nanowebs have shown great potentials specifically for environmental applications such as membranes/filters for air, liquid and solid filtration, water purification, personal respiratory systems, etc $[5,6]$.

Electrospinning of nanofibrous structures from functional materials is particularly important for filtration purposes since, specific filtering functionalities can be obtained from such materials along with high surface area and porous

Tamer Uyar

tamer@unam.bilkent.edu.tr

1 Institute of Materials Science \& Nanotechnology, UNAM-National Nanotechnology Research Center, Bilkent University, 06800 Ankara, Turkey characteristics. For instance, cyclodextrins (CD) which are produced from enzymatic degradation of starch, are cyclic oligosaccharides which have toroid-shaped molecular structure that can entrap variety of molecules in their cavity by forming non-covalent host-guest inclusion complexes [7, 8]. Along with numerous application areas of $\mathrm{CD}$ in pharmaceuticals, foods, cosmetics, home/personal care, textiles, etc $[7,8], \mathrm{CD}$ molecules are also quite applicable in filtration/ separation/purification systems due to their inclusion complexes capability with numerous pollutant molecules [9-11]. There are three native $\mathrm{CD}$ types; $\alpha-\mathrm{CD}, \beta-\mathrm{CD}$ and $\gamma-\mathrm{CD}$ which are classified according to number of the glucopyranose unit having six, seven, eight units in their molecular structure, respectively [7].

In our recent studies, by combining the high surface area of electrospun nanofibers and the host-guest inclusion complexation capability of $\mathrm{CD}$, we have performed cyclodextrin functionalization of electrospun polymeric nanofibers which can be used as molecular filters for water filtration $[12,13]$ or removal of volatile organic compounds (VOC) for air filtration [14-16]. Yet, the drawback of this approach was the presence of limited weight $\%$ of the $\mathrm{CD}$ on the fiber surface when nanofibers were electrospun from physical mixture of polymer/CD. Hence, given that nanofibers fully composed of $\mathrm{CD}$ are more attractive, we have firstly reported 
the electrospinning of nanofibers purely from chemically modified CD molecules from their highly concentrated aqueous solution without using a carrier polymeric matrix [17]. Although, electrospinning of nanofibers from native $\mathrm{CD}$ without using polymeric matrix are more challenging due to their low water solubility, we were also able to perform the electrospinning of nanofibers from native $\mathrm{CD}(\alpha-\mathrm{CD}$, $\beta$-CD) [18] using highly basic condition and we electrospun $\gamma$-CD nanofibers from highly concentrated solution of water/organic solvent mixture [19]. CD can self-assemble and form considerable aggregates via intermolecular hydrogen bonding in their concentrated solutions, so, this helps the electrospinning of nanofibers from highly concentrated CD solutions. At the same time, electrospinning of $\mathrm{CD}$ process requires a tedious optimization since $\mathrm{CD}$ molecules aggregate with time which the solution properties (i.e. solution viscosity) changes and therefore this makes the electrospinning of CD solutions much more challenging when compared to electrospinning of polymeric solutions. In this study, as an alternative approach, we mixed the native $\mathrm{CD}$ (i.e. $\gamma-\mathrm{CD}$ ) with a minimal amount of high molecular weight polymer carrier matrix (polyethylene oxide (PEO)) to stabilize the property (i.e. solution viscosity) of low concentrated $\mathrm{CD}$ solutions during the electrospinning for the uniform nanofiber formation. The different ratios of PEO: $\gamma$-CD (1:20\%, 2:20\%, 3:20\% and 1:40\%, with respect to solvent) were used to optimize the electrospinning of uniform nanofibers from aqueous solutions of $\mathrm{PEO} / \gamma-\mathrm{CD}$. After that, the PEO carrier matrix was selectively removed by simple washing procedure, at the end $\gamma$-CD short-nanofibers were obtained. The $\gamma$-CD short-nanofibers were tested for the removal of one of the most common VOC molecule; aniline. VOC are already considered as one of most hazardous classes of air pollutants, and aniline is one of the well-known types of these VOC with its highly toxic and carcinogenic characteristic.
Here, to facilitate the electrospinning process of $\gamma$-CD nanofibers, a very minimal amount of sacrificial polymeric matrix was used to obtain uniform and bead-free electrospun nanofibers. Afterwards, $\gamma$-CD short-nanofibers were obtained by removal of sacrificial polymeric matrix by simple washing procedure. The short $\gamma$-CD nanofibers were used for the removal of VOC from the surroundings owing to inclusion complexation capability of CD structure. The nanofibrous web composed of short $\gamma$-CD nanofibers demonstrated quite effective removal performance by encapsulating model VOC molecule; aniline from the surroundings. In short, our results indicated that $\mathrm{CD}$ based nanofibrous structures can be quite appropriate for air filtration due to their non-covalent inclusion complexation capability with variety of VOC molecules.

\section{Experimental}

The polyethylene oxide (PEO) $\left(\mathrm{M}_{\mathrm{v}} \sim 900.000\right.$, powder, Sigma Aldrich), chloroform (extra pure, Sigma Aldrich), aniline (99\%, Sigma Aldrich) and deuterated dimethylsulfoxide (d6-DMSO, deuteration degree min. $99.8 \%$ for NMR spectroscopy, Merck) were purchased commercially. The $\gamma$-cyclodextrin $\left(\gamma\right.$-CD, CAVAMAX ${ }^{\circledR}$ W8) was a gift from Wacker Chemie AG (Germany). The materials were used without any purification. Homogenous solutions of polymer were prepared by dissolving PEO in water at $1,2,3 \%(\mathrm{w} / \mathrm{v})$ concentrations. Then $\gamma$-CD was added to these PEO solutions at $20 \%(\mathrm{w} / \mathrm{v})$ concentration, besides $1 \% \mathrm{PEO} / 40 \% \gamma$-CD solutions was prepared. The clear solutions were electrospun using metallic needle syringe (inner diameter $=0.45 \mathrm{~mm}$ ) which was fixed horizontally on the syringe pump (Model: SP 101IZ, WPI). Matsusada Precision (AU Series, Japan) was used as a high voltage power supply. The electrospinning parameters were as follows:

Table 1 Properties of the electrospinning solutions and the resulting nanofibers

\begin{tabular}{|c|c|c|c|c|c|c|}
\hline Solution & $\% \operatorname{PEO}(\mathrm{w} / \mathrm{v})^{\mathrm{a}}$ & $\% \gamma-C D(w / v)^{a}$ & Viscosity $(\mathrm{Pa} \mathrm{s})^{\mathrm{b}}$ & $\begin{array}{l}\text { Conductivity } \\
(\mu \mathrm{S} / \mathrm{cm})\end{array}$ & Fiber morphology & $\begin{array}{l}\text { Average fiber } \\
\text { diameter } \\
(\mathrm{nm})^{\mathrm{c}}\end{array}$ \\
\hline $1 \% \mathrm{PEO}$ & 1 & - & 0.0123 & 130 & Beaded nanofiber & - \\
\hline $2 \% \mathrm{PEO}$ & 2 & - & 0.0505 & 223 & Beaded nanofiber & - \\
\hline $3 \% \mathrm{PEO}$ & 3 & - & 0.1540 & 292 & Bead-free nanofibers & $145 \pm 35$ \\
\hline $1 \% \mathrm{PEO} / \% 20 \gamma-\mathrm{CD}$ & 1 & 20 & 0.0166 & 92 & Beaded nanofiber & - \\
\hline $2 \% \mathrm{PEO} / \% 20 \gamma-\mathrm{CD}$ & 2 & 20 & 0.2110 & 134 & Bead-free nanofibers & $715 \pm 115$ \\
\hline $3 \% \mathrm{PEO} / \% 20 \gamma-\mathrm{CD}$ & 3 & 20 & 0.4190 & 171 & Bead-free nanofibers & $590 \pm 125$ \\
\hline $1 \% \mathrm{PEO} / \% 40 \gamma-\mathrm{CD}$ & 1 & 40 & 0.0322 & 76 & Bead-free nanofibers & $540 \pm 130$ \\
\hline
\end{tabular}

$\mathrm{a} \%(\mathrm{w} / \mathrm{v})$ is with respect to solvent (water)

${ }^{\mathrm{b}}$ Steady values under a constant shear rate of 100 1/s (from 12 data points, $5 \mathrm{~s}$ time intervals)

${ }^{\mathrm{c}}$ Calculated from $\sim 100$ fibers 
applied voltage $=10-15 \mathrm{kV}$, feed rate $=0.5-1 \mathrm{ml} / \mathrm{h}$ and tipto-collector distance $=10-15 \mathrm{~cm}$. Electrospun nanofibers were deposited on a grounded stationary cylindrical metal collector covered by a piece of aluminum foil. The electrospinning process was carried out in enclosed Plexiglas box and at $22{ }^{\circ} \mathrm{C}$ at $30 \%$ relative humidity. To remove the PEO part from nanofibers, $2 \% \mathrm{PEO} / 20 \% \gamma-\mathrm{CD}, 3 \% \mathrm{PEO} / 20 \% \gamma-\mathrm{CD}$ and $1 \% \mathrm{PEO} / 40 \% \gamma-\mathrm{CD}$ nanofibers having uniform morphology were washed for $\sim 3 \mathrm{~h}$ in chloroform. Then washed nanowebs were kept in vacuum oven over night at $60{ }^{\circ} \mathrm{C}$.
The viscosity measurements were performed at a constant shear rate of $100 \mathrm{1} / \mathrm{s}$ at $22{ }^{\circ} \mathrm{C}$ using Anton Paar Physica MCR 301 rheometer. The conductivity of the solutions was measured with Multiparameter meter InoLab ${ }^{\circledR}$ Multi 720 (WTW) at $22{ }^{\circ} \mathrm{C}$. The morphology and the diameter of the nanofibers were investigated using scanning electron microscope (SEM) (FEI-Quanta 200 FEG). Samples were coated with $5 \mathrm{~nm} \mathrm{Au} / \mathrm{Pd}$ prior to SEM imaging. The nanofibers diameter ranges were determined from the SEM images and around 100 fibers were analyzed. The X-ray diffraction
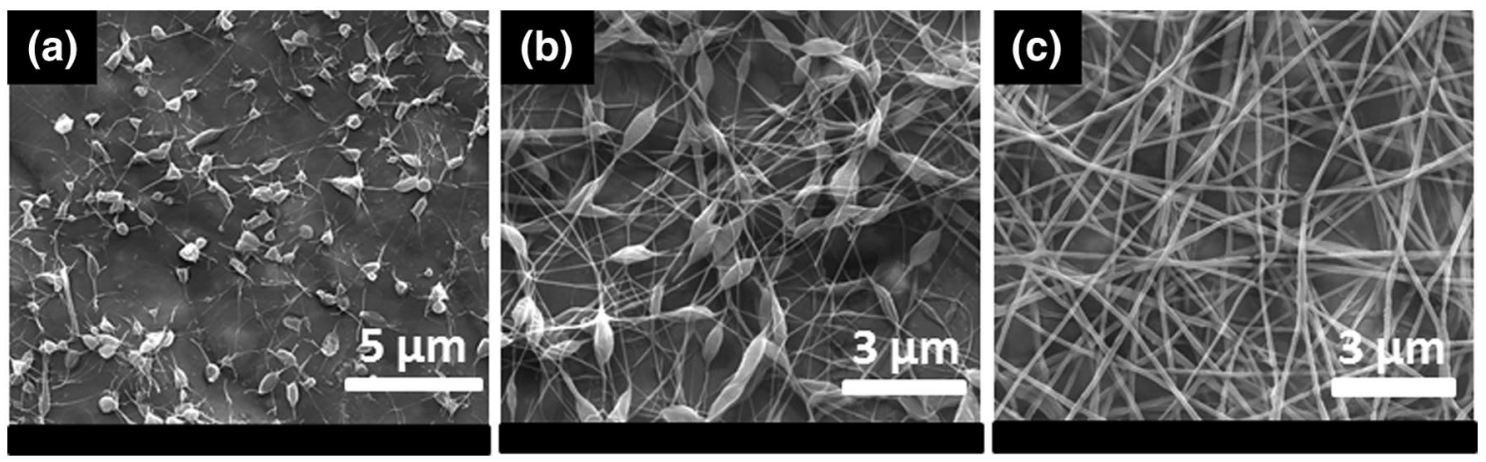

Fig. 1 SEM images of the electrospun nanofibers from a 1\%PEO, b 2\%PEO, c 3\%PEO (w/v) polymer concentrations

Fig. 2 The representative SEM images of the electrospun nanofibers from a $1 \% \mathrm{PEO} / 20 \% \gamma-\mathrm{CD}$, b $2 \% \mathrm{PEO} / 20 \% \gamma-\mathrm{CD}$, c $3 \% \mathrm{PEO} / 20 \% \gamma-\mathrm{CD}$ and $\mathbf{d}$ $1 \% \mathrm{PEO} / 40 \% \gamma-\mathrm{CD}$
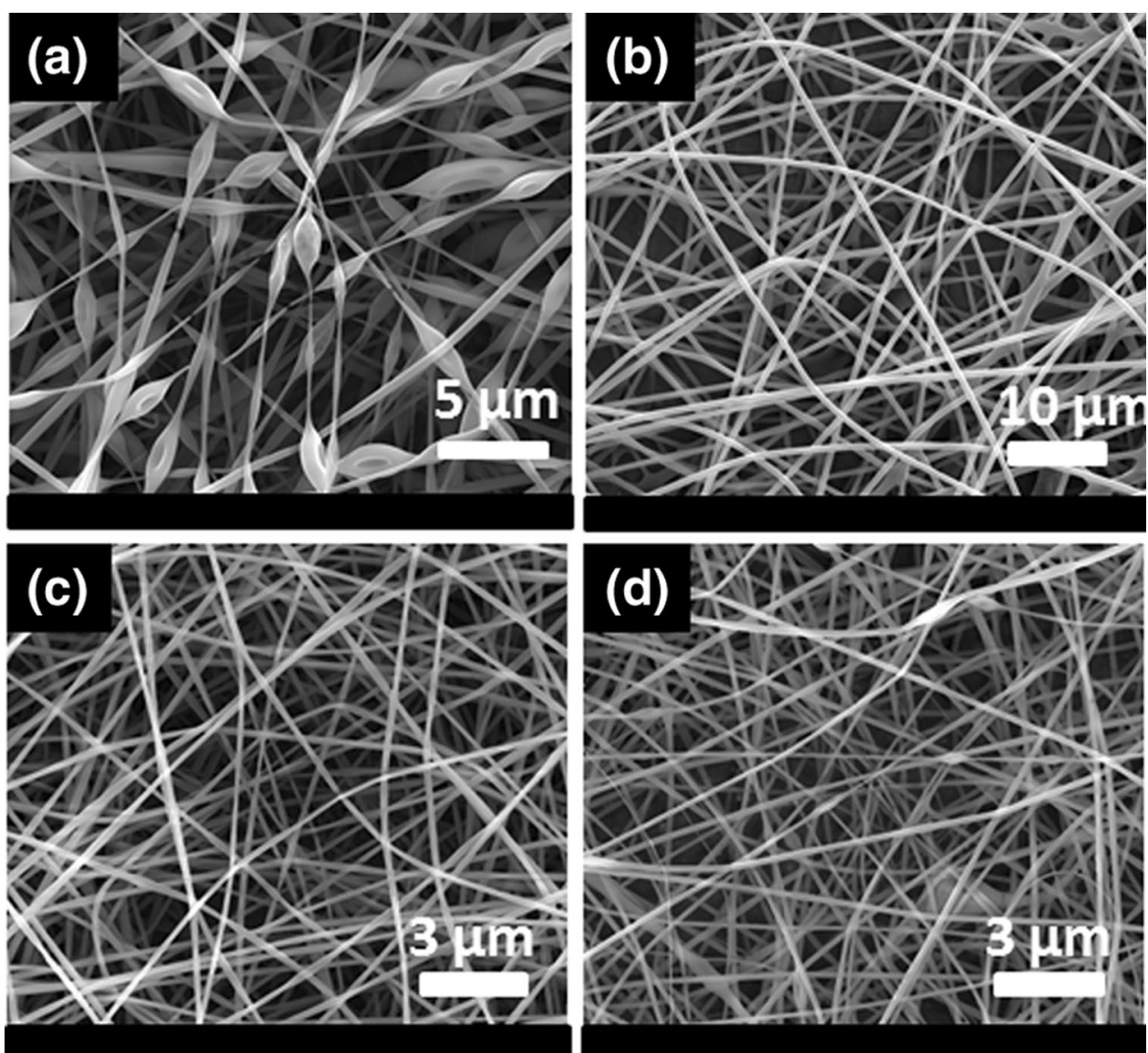
(XRD) (PANalytical X'Pert powder diffractometer) data of the samples were recorded using $\mathrm{Cu} \mathrm{K} \alpha$ radiation in a range of $2 \theta=5^{\circ}-30^{\circ}$. The molecular filtration performance of $\gamma$-CD short-nanofibers, obtained by the washing off $1 \% \mathrm{PEO} / 40 \% \gamma$-CD nanofibers, was evaluated by entrapping aniline vapor. For comparison, the entrapment test was also performed for as-received $\gamma$-CD powder. For this, $10 \mathrm{ml}$ of aniline was put into glass Petri dishes and located at the bottom of the desiccator (30 cm (diameter) and $30 \mathrm{~cm}$ (height)). Then, samples were located into the sealed desiccator. Samples were exposed aniline vapor for $24 \mathrm{~h}$, afterwards, they were taken out of the desiccators and kept into suction hood for a while to remove aniline molecules that were just adsorbed and could not form inclusion complex with CD molecules. The experiments were performed in triplicate. The proton magnetic resonance $\left({ }^{1} \mathrm{H}\right.$ NMR) (Bruker DPX $400 \mathrm{MHz}$ ) was used to examine the encapsulated amount of aniline. The samples were dissolved in d6-DMSO at the $20 \mathrm{~g} / \mathrm{L}$ concentration. The spectra were recorded at $400 \mathrm{MHz}$ and at 16 total scan. Integration of the chemical shifts $(\delta)$ given in parts per million (ppm) of the samples was calculated using Mestrenova software. The molar ratios were calculated taking account the integration of aniline aromatic peaks (6.7 and 7.1 ppm) and the $\gamma$-CD's characteristic peak at about $4.8 \mathrm{ppm}$ for $d 6$-DMSO system.

\section{Results and discussion}

In our earlier reports, we have successfully performed electrospinning of nanofibers purely from chemically modified CD [17] and native CD [18, 19] molecules without using a carrier polymeric matrix. Yet, electrospinning of CD solutions is much more challenging when compared to electrospinning of polymeric solutions since solution properties (i.e. solution viscosity) do change with time due to aggregation of CD molecules in their highly concentrated solution (i.e. $160 \%, \mathrm{w} / \mathrm{v}$ ) and therefore tedious optimization is required for the electrospinning process for $\mathrm{CD}$ molecules.

Here, we took an alternative approach to obtain $\gamma$-CD nanofibers using a sacrificial polymeric matrix (i.e. PEO) for the electrospinning. We electrospun the nanofibers from a common aqueous solution of $\mathrm{PEO} / \gamma-\mathrm{CD}$ where we used a minimal amount of high molecular weight PEO carrier matrix for trouble-free electrospinning process. Table 1 summarizes the solution compositions of the PEO and $\mathrm{PEO} / \gamma-\mathrm{CD}$ and solution properties (i.e. viscosity and
Fig. 3 The representative SEM images of the $\gamma$-CD short-nanofibers which obtained after washing off from a $2 \% \mathrm{PEO} / 20 \% \gamma-\mathrm{CD}$, b $3 \% \mathrm{PEO} / 20 \% \gamma-\mathrm{CD}$, c $1 \% \mathrm{PEO} / 40 \% \gamma$-CD nanofibers and $\mathbf{d}$ cage $\gamma$-CD
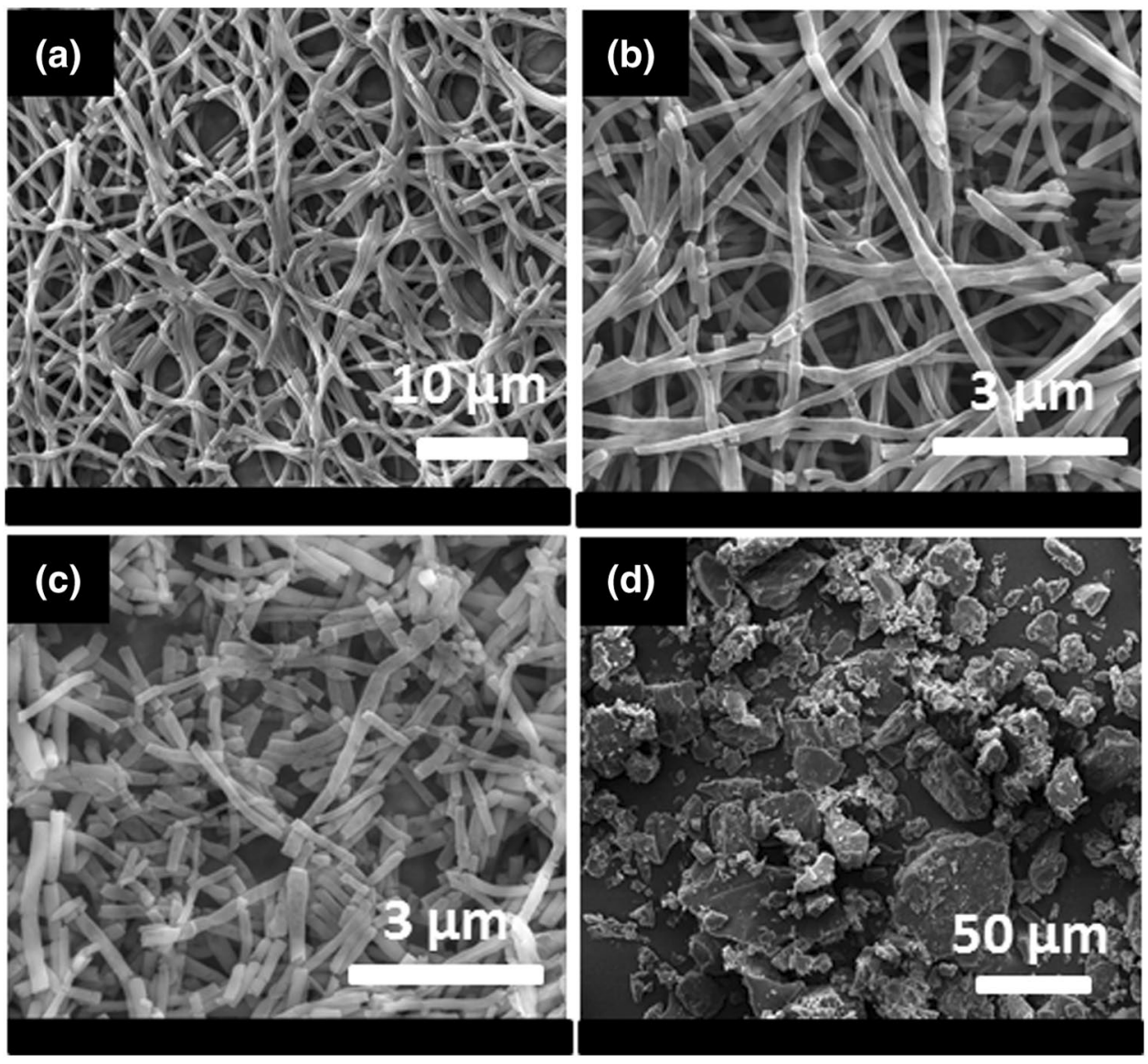
conductivity) and the morphology and average fiber diameter (AFD) of the resulting electrospun nanofibers. As an initial study, we optimized the electrospinning of pure PEO aqueous solutions having different concentration (1,2 and $3 \%(\mathrm{w} / \mathrm{v}))$. Figure 1 shows the SEM images where $1 \%(\mathrm{w} / \mathrm{v})$ PEO solution only yielded beads without any continuous fiber formation due to the very low solution viscosity (0.0123 Pa s). The electrospinning of $2 \%$ (w/v) PEO solution resulted in beaded-fiber morphology due to low solution viscosity ( $0.0505 \mathrm{~Pa} \mathrm{~s})$, but, once the concentration of PEO solution was increased to $3 \%(\mathrm{w} / \mathrm{v})(0.1540 \mathrm{~Pa}$ s), bead-free nanofibers were obtained with an AFD of $145 \pm 35 \mathrm{~nm}$. This is a well-known phenomena in the literature where high concentration/viscosity is required to produce bead-free PEO nanofibers [20]. It is also noted that the solution conductivity of the PEO solution has increased as the polymer concentration increased which easy up the electrospinning process where the jet is more elongated and stretched to form a beadfree fibers.

The aqueous solutions having different ratios of PEO: $\gamma$-CD $(1: 20 \%, 2: 20 \%, 3: 20 \%$ and 1:40\%, with respect to solvent) were used to optimize the electrospinning of uniform nanofibers from PEO/ $\gamma$-CD systems (Fig. 2). The electrospinning of $1 \% \mathrm{PEO} / 20 \% \gamma-\mathrm{CD}$ solution yielded beaded nanofibers (Fig. 2a) whereas 1\%PEO/40\% $\gamma$-CD resulted in bead-free uniform nanofibers with AFD of $540 \pm 130 \mathrm{~nm}$ (Fig. 2d). Although the 1\% (w/v) PEO solution yielded only beads (Fig. 1a), the presence of $\gamma$-CD facilitated the fiber formation. Similarly, $2 \% \mathrm{PEO} / 20 \% \gamma$-CD system yielded bead-free nanofibers (Fig. 2b) with AFD of $715 \pm 115 \mathrm{~nm}$ whereas $2 \%(w / v)$ PEO solution yielded beaded fibers (Fig. 1b). The electrospinning of 3\% PEO/20\% $\gamma$-CD solution yielded bead-free uniform nanofibers (Fig. 2c) with AFD of $590 \pm 125 \mathrm{~nm}$ similar to 3\% (w/v) PEO solution but the fibers are thicker than the ones obtained from $3 \%(\mathrm{w} / \mathrm{v})$ PEO solution (Fig. 1c). In general, we observed that the presence of $\gamma$-CD caused an increase in solution viscosity and decrease in solution conductivity. The PEO polymeric matrix helps the continuous electrospinning process and higher amount of $\gamma$-CD facilitated the uniform and beadfree fiber formation mostly due to high solution viscosity. These results correlates with our previous reports [20, 21] and other literature [22] where the presence of CD assist the formation of bead-free fibers from lower polymer solutions.

Here, our aim was to obtain nanofibers only from $\gamma$-CD and that's why we intend to use minimal amount of PEO matrix. As a further step, we selectively remove PEO matrix to obtain $\gamma$-CD fibers only which the $\mathrm{CD}$ cavities further will be used for the VOC removal by inclusion complexation. Once the PEO matrix was removed, we obtained shortnanofibers of $\gamma$-CD (Fig. 3c), the continuous fibers were breakdown into short-nanofibers since the long polymeric chains were keeping them intact.
XRD studies were performed for the $\mathrm{PEO} / \gamma-\mathrm{CD}$ electrospun nanofibers to investigate whether $\gamma$-CD molecules were present in the fiber matrix as crystalline aggregates or not. The as-received $\gamma$-CD is in white powder form and is crystalline material having crystals with irregular shape in few microns size (Fig. 3d) with "cage-type" packing structures (Fig. 4a) [19]. PEO is a semi-crystalline polymer with salient diffraction peaks at $2 \theta=19^{\circ}$ and $23^{\circ}$ (Fig. 4a). Interestingly, the XRD patterns for the electrospun $\mathrm{PEO} / \gamma-\mathrm{CD}$ nanofibers $(1 \% \mathrm{PEO} / 40 \% \gamma-\mathrm{CD}, 2 \% \mathrm{PEO} / 20 \% \gamma-\mathrm{CD}$ and $3 \% \mathrm{PEO} / 20 \% \gamma-\mathrm{CD}$ ) have broad halo (Fig. $4 \mathrm{a}$ ) suggesting an amorphous structure for both $\gamma$-CD and PEO. Even the quantity of $\gamma$-CD was quite high (up to 10-40 times with respect to polymeric matrix) in $\mathrm{PEO} / \gamma-\mathrm{CD}$ nanofibers, no distinct
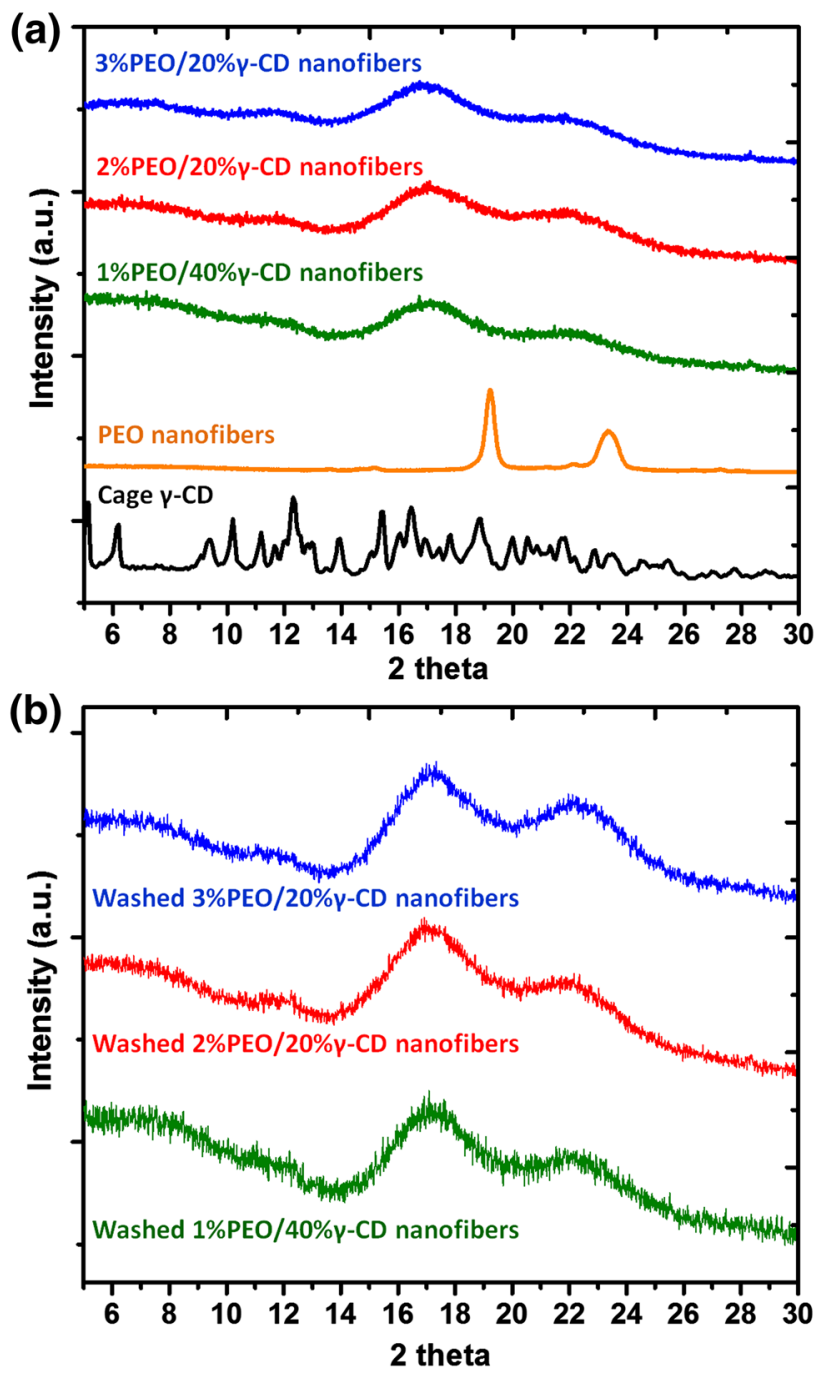

Fig. 4 XRD patterns of a as-received $\gamma$-CD powder with cagetype packing, PEO nanofibers, $1 \% \mathrm{PEO} / 40 \% \gamma-\mathrm{CD}$ nanofibers, $2 \% \mathrm{PEO} / 20 \% \gamma-\mathrm{CD}$ nanofibers, $3 \% \mathrm{PEO} / 20 \% \gamma-\mathrm{CD}$ nanofibers and $\mathbf{b}$ $\gamma$-CD short-nanofibers obtained by washing off $1 \% \mathrm{PEO} / 40 \% \gamma-\mathrm{CD}$, $2 \% \mathrm{PEO} / 20 \% \gamma-\mathrm{CD}$ and $3 \% \mathrm{PEO} / 20 \% \gamma-\mathrm{CD}$ nanofibers 
characteristic peaks for the $\gamma$-CD crystals were observed which suggested that the $\gamma$-CD molecules were distributed homogeneously in the fiber matrix without forming any phase separated crystal aggregates. We have also considered the possibility of complexation between PEO chains and $\gamma$-CD cavities, yet, this possibility was ruled out since in case a inclusion complexation, $\gamma$-CD would from "channel-type" crystalline structure [23]. After removal of PEO by selective washing with chloroform, $\gamma$-CD short-nanofibers still did not have shown any particular diffraction peaks confirming the preservation of their amorphous packing of $\gamma$-CD molecules. In our previous study, similar amorphous characteristic was also observed for the $\gamma$-CD nanofibers electrospun from dimethyl sulfoxide/water mixture without the use of polymeric matrix [19]. Actually, having amorphous $\gamma$-CD would be advantage for the filtration purposes when compared to as-received "cage-type" packing where CD cavities are block with each other [19]. VOC are groups of organic molecules which are considered as one of most hazardous classes of air pollutants. Aniline is one of the well-known VOC type with its highly toxic and carcinogenic characteristic. Hence, it is important to develop new filtering materials for the efficient removal of VOC from the environment [14, $16,19]$. Here, the VOC removal capability/capacity of the $\gamma$-CD short-nanofibers was tested by exposing the sample to VOC vapor (i.e. aniline) in a desiccator. The as-received $\gamma$-CD powder was also tested at the same time for comparison. It is expected that the entrapment of aniline vapor is possible through inclusion complexation by available $\gamma$-CD cavity. After $24 \mathrm{~h}$ exposure to aniline vapor, the $\gamma$-CD shortnanofibers and as-received $\gamma$-CD powder were separately dissolved in $d 6$-DMSO and the presence and molar ratio of aniline to $\gamma$-CD was determined by ${ }^{1} \mathrm{H}$-NMR spectroscopy (Fig. 5). ${ }^{1} \mathrm{H}$-NMR results revealed that the $\gamma$-CD nanofibrous sample effectively entrapped aniline whereas as-received $\gamma$-CD powder could not entrap the aniline from the surrounding. The molar ratio of aniline: $\gamma-C D$ was calculated as 0.99:1 \pm 0.07 for $\gamma$-CD nanofibrous sample suggesting that each $\gamma$-CD cavity host one aniline molecule, but, we couldn't record any aniline peak in ${ }^{1} \mathrm{H}-\mathrm{NMR}$ spectrum of the as-received $\gamma$-CD powder exposed to aniline vapor. In the case of $\gamma$-CD nanofibrous sample, CD molecules were amorphous without forming crystalline packing; hence, it is likely that the $\gamma$-CD cavity was accessible for aniline for the inclusion complexation. In contrast, as-received $\gamma$-CD powder has a cage-type crystal packing where each CD cavity is blocked by the neighboring $\mathrm{CD}$ molecule and therefore $\mathrm{CD}$ cavity could not be available for the entrapment of aniline. The aniline removal capability and capacity by the $\gamma$-CD short-nanofibers is very much similar to our previous study where electrospun $\gamma$-CD nanofiber from polymer-free systems showed the similar aniline removal performance [19]. In brief, the use of small amount of sacrificial polymeric matrix facilitates the electrospinning process and $\gamma$-CD with nanofibrous morphology can be obtained by simple washing off polymeric matrix. Our findings suggest that electrospun $\gamma$-CD short-nanofibers can be applicable in air filtration for the removal of VOC.

\section{Conclusion}

The electrospinning of $\gamma$-CD/PEO systems $(1 \% \mathrm{PEO} / 20 \% \gamma-\mathrm{CD}, 2 \% \mathrm{PEO} / 20 \% \gamma-\mathrm{CD}, 3 \% \mathrm{PEO} / 20 \% \gamma-\mathrm{CD}$
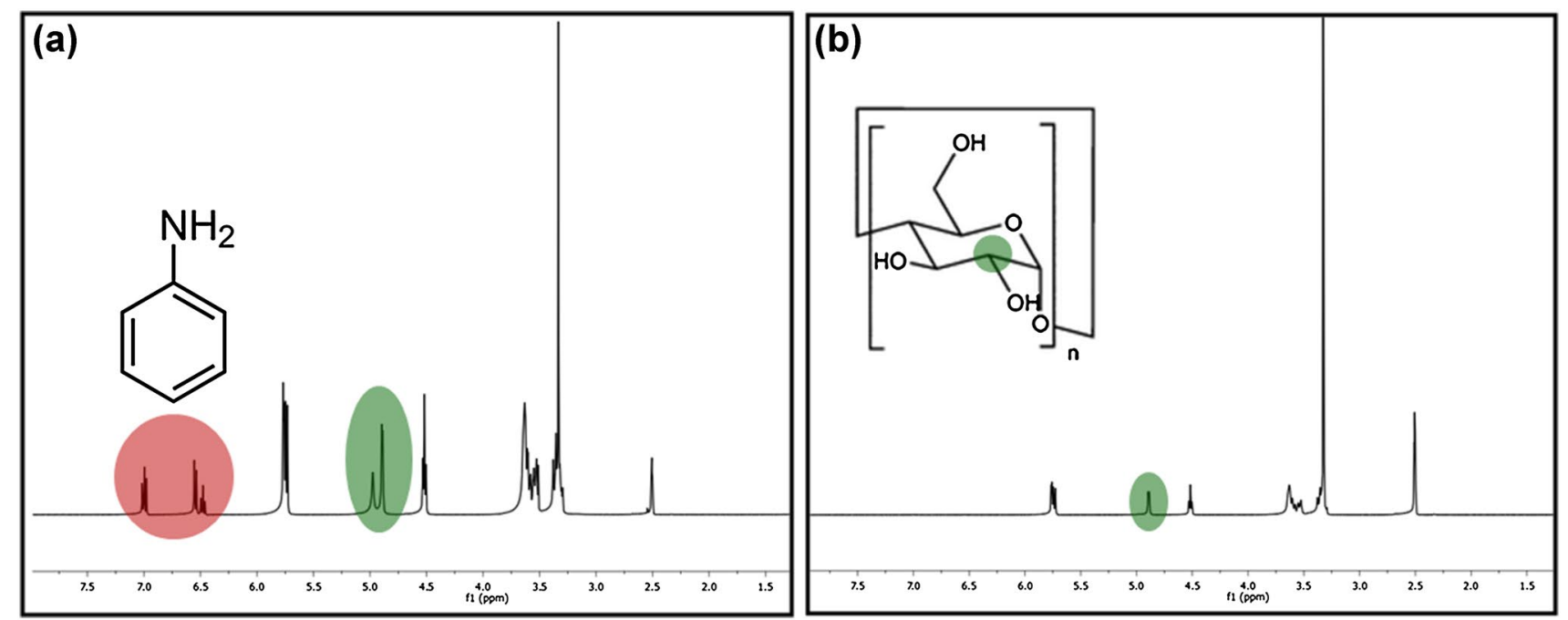

Fig. $5{ }^{1} \mathrm{H}$-NMR spectra of a $\gamma$-CD short nanofibers (obtained from $1 \% \mathrm{PEO} / 40 \% \gamma$-CD nanofibers) and $\mathbf{b}$ cage $\gamma$-CD powder after aniline entrapment test 
and $1 \% \mathrm{PEO} / 40 \% \gamma-\mathrm{CD}$ ) was performed using a minimal amount of PEO polymeric matrix. Once, the uniform and bead-free nanofibers were electrospun from $\gamma$-CD/PEO systems, PEO sacrificial matrix was selectively removed by simple washing procedure, and then, $\gamma$-CD short-nanofibers were obtained. Although the as-received $\gamma$-CD is cage-type crystalline structure, XRD studies revealed that the electrospun $\gamma$-CD short-nanofibers were amorphous without showing any crystalline packing of CD molecules. As a proof-ofconcept study, we have explored the molecular entrapment capability of $\gamma$-CD short-nanofibers for the removal of VOC (i.e. aniline). $\gamma$-CD short-nanofibers were quite successful for entrapping of aniline $(\sim 1: 1$ molar ratio of $\gamma$-CD:aniline $)$ whereas as-received $\gamma$-CD powder could not show any entrapment capability for aniline. In brief, electrospun $\gamma$-CD nanofibrous structures can be quite applicable as filtering material for air filtration due to their inclusion complexation capability with VOC.

Acknowledgements T. U. thanks to Turkish Academy of SciencesOutstanding Young Scientists Award Program (TUBA-GEBIP) for partial funding. A. C. thanks TUBITAK-BIDEB for the PhD scholarship.

\section{References}

1. Greiner, A., Wendorff, J.H., Agarwal, S.: Electrospinning: Materials, Processing, and Applications. Wiley, Weinheim (2012)

2. Uyar, T., Kny, E.: Electrospun Materials for Tissue Engineering and Biomedical Applications: Research, Design and Commercialization. Elsevier, Cambridge (2017)

3. Sahay, R., Kumar, P.S., Sridhar, R., Sundaramurthy, J., Venugopal, J., Mhaisalkar, S.G., Ramakrishna, S.: Electrospun composite nanofibers and their multifaceted applications. J. Mater. Chem. 22, 12953-12971 (2012)

4. Noruzi, M.: Electrospun nanofibres in agriculture and the food industry: a review. J. Sci. Food Agr. 96, 4663-4678 (2016)

5. Thavasi, V., Singh, G., Ramakrishna, S.: Electrospun nanofibers in energy and environmental applications. Energy Environ. Sci. 1, 205-221 (2008)

6. Sundarrajan, S., Tan, K.L., Lim, S.H., Ramakrishna, S.: Electrospun nanofibers for air filtration applications. Procedia Eng. 75, 159-163 (2014)

7. Szejtli, J.: Introduction and general overview of cyclodextrin chemistry. Chem. Rev. 98, 1743-1754 (1998)

8. Hedges, A.R.: Industrial applications of cyclodextrins. Chem. Rev. 98, 2035-2044 (1998)
9. Landy, D., Mallard, I., Ponchel, A., Monflier, E., Fourmentin, S.: Remediation technologies using cyclodextrins: an overview. Environ. Chem. Lett. 10, 225-237 (2012)

10. El Idrissi, M., Bacca, A.E.M., Frascari, D., Corvini, P.F.X., Shahgaldian, P.: Cyclodextrin-based polymeric materials for the specific recovery of polyphenolic compounds through supramolecular host-guest interactions. J. Incl. Phenom. Macrocycl. Chem. 88, $1-8(2017)$

11. Carvalho, L.B., Carvalho, T.G., Magriotis, Z.M., de Castro Ramalho, T., Pinto, L.D.M.A.: Cyclodextrin/silica hybrid adsorbent for removal of methylene blue in aqueous media. J. Incl. Phenom. Macrocycl. Chem. 78, 77-87 (2014)

12. Uyar, T., Havelund, R., Nur, Y., Hacaloglu, J., Besenbacher, F., Kingshott, P.: Molecular filters based on cyclodextrin functionalized electrospun fibers. J. Membr. Sci. 332, 129-137 (2009)

13. Uyar, T., Havelund, R., Hacaloglu, J., Besenbacher, F., Kingshott, P.: Functional electrospun polystyrene nanofibers incorporating $\alpha-, \beta-$, and $\gamma$-cyclodextrins: comparison of molecular filter performance. ACS Nano 4, 5121-5130 (2010)

14. Uyar, T., Havelund, R., Nur, Y., Balan, A., Hacaloglu, J., Toppare, L., Flemming, B., Kingshott, P.: Cyclodextrin functionalized poly (methyl methacrylate)(PMMA) electrospun nanofibers for organic vapors waste treatment. J. Membr. Sci. 365, 409-417 (2010)

15. Kayaci, F., Sen, H.S., Durgun, E., Uyar, T.: Electrospun nylon 6, 6 nanofibers functionalized with cyclodextrins for removal of toluene vapor. J. Appl. Polym. Sci. 132, 18 (2015)

16. Kayaci, F., Uyar, T.: Electrospun polyester/cyclodextrin nanofibers for entrapment of volatile organic compounds. Polym. Eng. Sci. 54, 2970-2978 (2014)

17. Celebioglu, A., Uyar, T.: Electrospinning of nanofibers from nonpolymeric systems: polymer-free nanofibers from cyclodextrin derivatives. Nanoscale. 4, 621-631 (2012)

18. Celebioglu, A., Uyar, T.: Electrospinning of nanofibers from nonpolymeric systems: electrospun nanofibers from native cyclodextrins. J. Colloid Interface Sci. 404, 1-7 (2013)

19. Celebioglu, A., Uyar, T.: Electrospun gamma-cyclodextrin $(\gamma-\mathrm{CD})$ nanofibers for the entrapment of volatile organic compounds. RSC Adv. 3, 22891-22895 (2013)

20. Uyar, T., Besenbacher, F.: Electrospinning of cyclodextrin functionalized polyethylene oxide (PEO) nanofibers. Eur. Polym. J. 45, 1032-1037 (2009)

21. Uyar, T., Havelund, R., Hacaloglu, J., Zhou, X., Besenbacher, F., Kingshott, P.: The formation and characterization of cyclodextrin functionalized polystyrene nanofibers produced by electrospinning. Nanotechnology. 20, 125605 (2009)

22. Burns, N.A., Burroughs, M.C., Gracz, H., Pritchard, C.Q., Brozena, A.H., Willoughby, J., Khan, S.A.: Cyclodextrin facilitated electrospun chitosan nanofibers. RSC Adv. 5, 7131-7137 (2015)

23. Uyar, T., El-Shafei, A., Wang, X., Hacaloglu, J., Tonelli, A.E.: The solid channel structure inclusion complex formed between guest styrene and host $\gamma$-cyclodextrin. J. Incl. Phenom. Macrocycl. Chem. 55, 109-121 (2006) 